\title{
Dynamic Similarity Kernel for Visual Recognition
}

\author{
Wang Yan, Qingshan Liu, Hanqing Lu, and Songde Ma \\ National Laboratory of Pattern Recognition \\ Institute of Automation, Chinese Academy of Sciences \\ P.O. Box 2728, Beijing, P.R. China, 100080 \\ \{wyan, qsliu, luhq, masd\}@nlpr.ia.ac.cn
}

\begin{abstract}
Inspired by studies of cognitive psychology, we proposed a new dynamic similarity kernel for visual recognition. This kernel has great consistency with human visual similarity judgement by incorporating the perceptual distance function. Moreover, this kernel can be seen as an extension of Gaussian kernel, and therefore can deal with nonlinear variations well like the traditional kernels. Experimental results on natural image classification and face recognition show its superior performance compared to other kernels.
\end{abstract}

\section{Introduction}

Researchers on computer vision and pattern recognition, regularly confront the problem of nonlinearity in high-dimensional data, such as variation in image due to the change of illumination and viewpoint. Generally speaking, there are two schemes to solve the problem. The first one is to extend the existing linear method by so-called kernel trick [1, such as extensions of Principal Component Analysis (PCA) [2] and Linear Discriminant Analysis (LDA) [3]. Recently, these kernel methods attract much attention and show promising performance in many applications, such as image retrieval [4,5,6], face recognition [7,8] and object recognition 9,10 . Using nonlinear functions to model or to transform the nonlinear data is the second solution, such as neural network [11] and manifold learning [12,13, 14]. We focus on the kernel methods in this paper.

The basic idea of the kernel methods is first mapping data into an implicit feature space with a nonlinear function, and then analyzing the image of data. Instead of explicitly computing the mapping, a kernel function is adopted to calculate the inner product of implicit feature vectors. This function determines the nonlinear mapping implicitly. If the solution of a linear method is able to be expressed by the inner products of its input samples, this method can be extended to the nonlinear version. The kernel trick is first introduced into Support Vector Machine (SVM) in [15. Similar to SVM, PCA and LDA are extended to their nonlinear forms, i.e. Kernel PCA (KPCA) 16 and Generalized Discriminant Analysis (GDA) [17,18. PCA generates a set of orthonormal projections which maximizes the covariance over all samples, while LDA seeks a linear transformation which maximizes the inter-class scatter and minimizes the intra-class 
scatter. Their nonlinear extensions, i.e. KPCA and GDA, do the same thing in feature space.

To measure (dis)similarity between images, Minkowski distance functions are commonly used, in which all coordinates of image feature vector are employed. But studies of cognitive psychology [19,20 show that this approach does not model human perception well, which actually infers overall similarity based on the aspects that are similar among the compared objects, rather than based on the dissimilar ones. From this point of view, Dynamic Partial Function (DPF) 21, 22 is proposed, which can be seen as an extension of Minkowski distance. By dynamically selecting features with minimum differences in a pairwise fashion, DPF works similarly to human process of similarity judgement. Extensive experiments in 21,22] show its effectiveness.

A modified kernel function based on geodesic distance [12] is proposed in [23], and experimental results show its promising performance. But it still has some difficulties modeling human perception, because the estimation of geodesic distance is based on Minkowski distance. In this paper, we present a new kernel based on DPF, which has great consistency with human visual similarity judgement. Moreover, this kernel can be seen as an extension of Gaussian kernel, and therefore can deal with nonlinear variations well like the traditional kernels. By using KPCA and GDA on Corel and FERET database, we test this kernel and compare it with other traditional kernels, such as Gaussian and polynomial kernels. The experimental results show that the proposed kernel outperforms the others.

The rest of this paper is organized as follows. In Section 2, we describe Dynamic Partial Function for measuring image similarity. Dynamic Similarity Kernel is proposed in Section 3. Section 4 presents the experimental results on natural image classification and face recognition, followed by conclusions in Section 5 .

\section{Dynamic Partial Function}

Minkowski distance is widely used for measuring similarity between images. Let $X=\left(x_{1}, x_{2}, \cdots, x_{n}\right)$ and $Y=\left(y_{1}, y_{2}, \cdots, y_{n}\right)$ be two image feature vectors, the distance under Minkowski metric is defined as

$$
D(X, Y)=\left(\sum_{i=1}^{n} \Delta d_{i}^{r}\right)^{\frac{1}{r}},
$$

where $\Delta d_{i}=\left|x_{i}-y_{i}\right|$, and $r$ is the norm factor. When we set $r=2$, the above is the well known Euclidean or L2 distance. When we set $r=1$, it is the City-block or L1 distance.

Studies of cognitive psychology 20] show that human perception of similarity is the process that determines the respects for measuring similarity. Human infers overall similarity based on the aspects that are similar among the compared objects, rather than based on the dissimilar ones. 21] verifies this notion by extensive experiments on natural image database. From this point of view, the 
similarity based on Minkowski distance which incorporates all aspects of the compared objects is questionable.

Dynamic Partial Function is proposed in 21,22, to solve the above problem. It is a modified version of Minkowski distance. For the sake of clarity, we first assume $\Delta d_{i}$ s to be ordered as $\Delta d_{1} \leq \Delta d_{2} \leq \cdots \leq \Delta d_{n}$. The DPF is defined as

$$
D P F(X, Y)=\left(\frac{1}{m} \sum_{i=1}^{m} \Delta d_{i}^{r}\right)^{\frac{1}{r}},
$$

where $m \leq n$ is the number of aspects activated in similarity measurement. Different from Minkowski distance, DPF dynamically selects the subset of features that are most similar for a given pair of images, and computes the similarity based on it. Hence, it works in a similar way as human visual perception system does. Empirical studies in [21] show its performance to be superior to other widely used distance functions, such as fractional function, histogram cosine distance function and Minkowski distance.

\section{Dynamic Similarity Kernel}

Kernel function is important for the kernel based methods. One can compute inner product in feature space efficiently by kernel function. Some kernels are based on Minkowski distance, such as Gaussian kernel and exponential kernel [24]. The former is defined as

$$
K(X, Y)=\exp \left(-\frac{\|X-Y\|^{2}}{n \sigma^{2}}\right),
$$

where $\sigma^{2}$ is the bandwidth, and $n$ is the dimensionality of $X$ and $Y$.

[23] substitutes geodesic distance for Euclidean distance in (3), and shows promising results. But the estimation of geodesic distance is based on Euclidean distance, and also employs all coordinates of the feature vector. So, it doesn't model human visual perception well, either.

To overcome this defect, we proposed a new kernel based on DPF as follows.

$$
K(X, Y)=\exp \left(-\frac{D P F^{2}(X, Y)}{\sigma^{2}}\right) .
$$

A kernel dot product can be seen as a similarity measure. The similarity measured by the proposed kernel is more accurately than Gaussian kernel, because of DPF's consistency with human perception. It dynamically activates the most similar aspects when evaluating the similarity of image pair. For its dynamic nature, we call it Dynamic Similarity Kernel (DSK for short). Since DPF is an extension of Minkowski distance, Gaussian kernel can be seen as a special case of DSK. We set $r=2$ for DPF in (44) for analogue with Gaussian kernel. 


\section{Experiments}

To examine the effectiveness of the proposed kernel, we compare it with other traditional kernels in some classic kernel methods, i.e. KPCA and GDA. The results are also compared with state-of-the-art. Two types of experiments are conducted. One is natural image classification, and the other is face recognition. They are both current hot topics in computer vision and pattern recognition.

\subsection{Natural Image Classification}

The WANG dataset 25,26] is commonly used in the literature to evaluate image retrieval and classification methods. It consists of 10 image categories, each of which contains 100 images. The themes of these categories are African people and villages, beach, buildings, buses, dinosaurs, elephants, flowers, horses, mountain and glaciers, and food.

Three types of features are used to represent image: color histogram, color moment and wavelet based texture. Color histogram is taken in HSV space with quantization of $8 \times 8=64$ bins on $\mathrm{H}$ and $\mathrm{S}$ channels. The first three moments from each of the three color channels are used as color moment. Mean and variance of each channel in a 4-level PWT decomposition form the wavelet based texture feature. In total, a 105-dimensional feature vector is extracted for each image. Each feature component is normalized such that the variance of which equals $\frac{1}{3}$.

We use a leave-one-out cross-validation as the testing protocol. That is, choose one image one time and use the rest 999 images as training set, then test the image left out. The classification accuracy is averaged on the whole dataset. Nearest neighbor classifier is used for classification.

There are two parameters in DSK, i.e. $m$ and $\sigma^{2}$. For the latter one, we first compute pair-wise distances for all images in the dataset, and then adaptively set $\sigma^{2}$ to the mean of the distances' square. Different values of $m$ are investigated, and the results are shown in Table 1. It can be seen that $m=80$ gives the best classification accuracy, which means about 25 dimensions are dissimilar aspects for pair-wise matching. We set $m=80$ in the following experiment.

Table 1. Accuracies (\%) on image classification with different $m$ values

\begin{tabular}{|c|c|c|c|c|c|c|c|c|c|}
\hline$m$ & 65 & 70 & 75 & 80 & 85 & 90 & 95 & 100 & 105 \\
\hline Accuracy & 82.8 & 84.0 & 84.7 & 85.4 & 85.2 & 84.4 & 84.0 & 83.7 & 78.3 \\
\hline
\end{tabular}

Next, we compare DSK with Gaussian kernel and polynomial kernels in KPCA. By investigating values in $[0.01,1]$, we find that Gaussian kernel with $\sigma^{2}=0.27$ achieves the best result of $78.3 \%$, and use this setting in the following comparison. There are two forms of polynomial kernels,

$$
K(X, Y)=(X \cdot Y)^{p}
$$


and

$$
K(X, Y)=(X \cdot Y+1)^{p},
$$

noted as PolyI and PolyII, respectively. We set $p=2$ for both because it gives the best results among $\{2,3,4,5\}$.

Nearest neighbor classifier using DPF directly has been investigated, too. It is found that $m=89$ gives the highest accuracy, i.e. $83.9 \%$.

In Fig. 1, the recognition accuracies of four kernels are plotted versus the number of dimensions used by the classifier, and the best result of direct DPF is also plotted as the baseline. Except for the accuracy of $85.4 \%$ achieved by DSK, the best results of Gaussian kernel, PolyI and PloyII are 78.3\%, 74.0\% and $75.5 \%$, respectively. Gaussian kernel is better than polynomial kernels. Because of similarity to human visual perception, DPF gets better result than traditional kernels. DSK outperforms all other kernels with large margins. It is noticeable that DSK can get even better result than DPF by incorporating Gaussian function to deal with nonlinearity. The best result in the literature is $84.1 \%$ [26, 27], while DSK breaks this record.

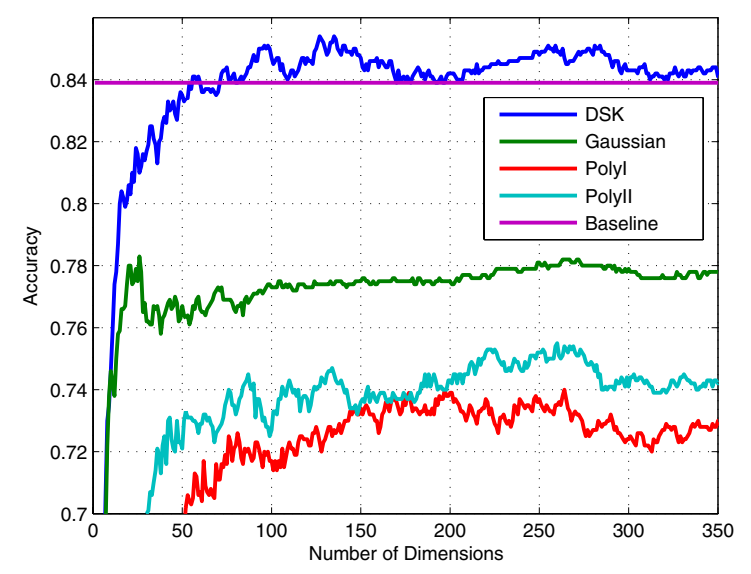

Fig. 1. Comparison of DSK, Gaussian kernel and polynomial kernels in image classification

\subsection{Face Recognition}

We conduct face recognition experiments on the FERET database. The experimental data include 1002 front view face images selected from the training CD, the FA set of 1196 subjects and the FB set of 1195 subjects. There's only one image per person in the FA and FB sets. Images from the training CD are used as training set. FA is used as gallery image set, and FB is taken as probe set. All images are normalized to $48 \times 54$ by eye locations, and histogram equalization is performed as preprocessing. The appearance of image, i.e. raw pixel value, is taken directly as the feature. Each feature component is normalized such that the variance of which equals 1 . 
GDA is adopted this time, for its popularity and excellent performance in face recognition 8, 28. We compare DSK with Gaussian kernel and PolyI, while PloyII is excluded according to 28. We set $\sigma^{2}=3.3$ in Gaussian kernel for the best performance, and set PolyI's parameter $p=2$ as in [28. $\sigma^{2}$ in DSK is set to 10 by investigating values from 0.1 to 20 . Classification results under different values of $m$ are listed in Table 2. It shows that $m / n=0.8$ gives the best accuracy. These settings are used in the following experiment.

Table 2. Accuracies (\%) on face recognition with different $m$ values

\begin{tabular}{|c|c|c|c|c|c|c|c|c|c|c|}
\hline$m / n$ & 0.55 & 0.60 & 0.65 & 0.70 & 0.75 & 0.80 & 0.85 & 0.90 & 0.95 & 1.00 \\
\hline Accuracy & 93.56 & 93.97 & 95.15 & 96.23 & 96.99 & 97.41 & 97.15 & 96.99 & 95.98 & 92.22 \\
\hline
\end{tabular}

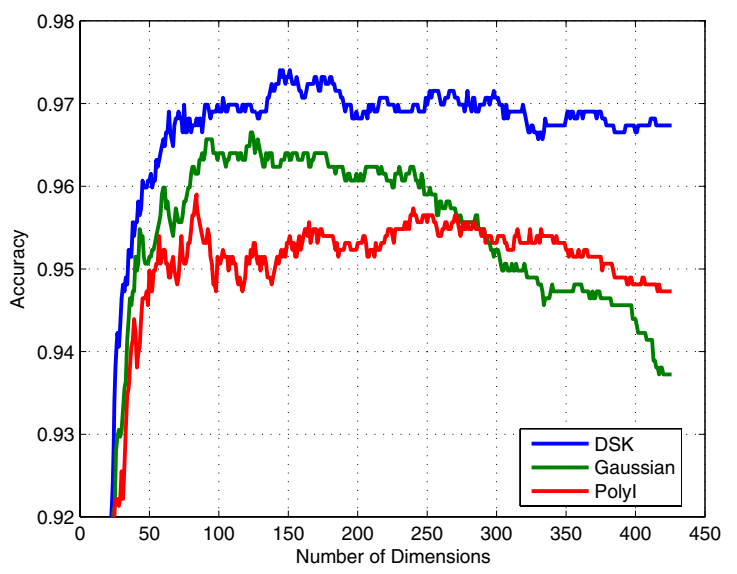

Fig. 2. Comparison of DSK, Gaussian kernel and polynomial kernels in face recognition

Fig. 2 shows the comparison of the three kernels. DSK gets the highest recognition rate of $97.41 \%$, and best result of Gaussian kernel and PolyI are $96.65 \%$ and $95.9 \%$, respectively. Once again, the superior performance implies that DSK is more consistent to human vision.

\section{Conclusion}

A kernel for visual recognition is proposed. By incorporating Dynamic Partial Function, the kernel has great consistency with human visual similarity judgement. Moreover, the kernel can be seen as an extension of Gaussian kernel. The adoption of Gaussian function enables its capability to deal with nonlinear variations effectively. Experimental results on natural image classification and face recognition show its superior performance compared to other classic kernels. 


\section{Acknowledgement}

This work is partially supported by the National Key Basic Research and Development Program (973) under Grant No. 2004CB318107, and the Natural Sciences Foundation of China under Grant No. 60405005 and 60121302.

\section{References}

1. Aizerman, M., Braverman, E., Rozonoer, L.: Theoretical foundations of the potential function method in pattern recognition learning. Automation and Remote Control 25 (1964) 821-837

2. Jolliffe, I.: Principal Component Analysis. Springer-Verlag, New York (1986)

3. Fisher, R.: The use of multiple measurements in taxonomic problems. Annals of Eugenics 7 (1936) 179-188

4. Wang, L., Gao, Y., Chan, K., Xue, P., Yau, W.: Retrieval with knowledge-driven kernel design: An approach to improving SVM-based CBIR with relevance feedback. In: Proc. of Int. Conf. Computer Vision. (2005)

5. Wu, G., Chang, E., Panda, N.: Formulating context-dependent similarity functions. In: Proc. of ACM Multimedia. (2005)

6. Yan, W., Liu, Q., Lu, H., Ma, S.: Multiple similarities based kernel subspace learning for image classification. In: Proc. of Asian Conf. Computer Vision. (2006)

7. Yang, M., Ahuja, N., Kriegman, D.: Face recognition using kernel eigenfaces. In: Proc. of Int. Conf. Image Processing. (2000)

8. Liu, Q., Lu, H., Ma, S.: Improving kernel fisher discriminant analysis for face recognition. IEEE Trans. on Circuits and Systems for Video Technology, Special Issue on Image and Video Based Biometrics 14 (2004) 42-49

9. Lyu, S.: Mercer kernels for object recognition with local features. In: Proc. of IEEE Computer Society Conf. Computer Vision and Pattern Recognition. (2005)

10. Grauman, K., Darrell, T.: The pyramid match kernel: Discriminative classification with sets of image features. In: Proc. of Int. Conf. Computer Vision. (2005)

11. Duda, R., Hart, P., Stork, D.: Pattern Classification. 2rd edn. John Wiley \& Sons, Inc (2001)

12. Tenenbaum, J., Silva, V., Langford, J.: A global geometric framework for nonlinear dimensionality reduction. Science 290 (2000) 2319-2323

13. Roweis, S., Saul, L.: Nonlinear dimensionality reduction by locally linear embedding. Science 290 (2000) 2323-2326

14. Belkin, M., Niyogi, P.: Laplacian eigenmaps and spectral techniques for embedding and clustering. In: Advances in Neural Information Processing System. Volume 13., Cambridge, MA, MIT Press (2001)

15. Vapnik, V.: Statistical Learning Theory. John Wiley \& Sons (1998)

16. Schölkopf, B., Smola, A., Müller, K.: Nonlinear component analysis as a kernel eigenvalue problem. Neural Computation 10 (1998) 1299-1319

17. Mika, S., Rätsch, G., Weston, J., Schölkopf, B., Müller, K.: Fisher discriminant analysis with kernels. In: Proc. of IEEE Neural Networks for Signal Processing Workshop. (1999)

18. Baudat, G., Anouar, F.: Generalized discriminant analysis using a kernel approach. Neural Computation 12 (2000) 2385-2404

19. Tversky, A.: Features of similarity. Psychological Rev. 84 (1977) 327-352 
20. Goldstone, R.: Similarity, interactive activation, and mapping. J. Experimental Psychology: Learning, Memory, and Cognition 20 (1994) 3-28

21. Li, B., Chang, E., Wu, Y.: Enhancing DPF for near-replica image recognition. ACM Multimedia J., special issue on content-based image retrieval 8 (2003) 512522

22. Qamra, A., Meng, Y., Chang, E.: Enhanced perceptual distance functions and indexing for image replica recognition. IEEE Trans. on Pattern Analysis and Machine Intelligence 27 (2005) 379-391

23. Yong, Q., Jie, Y.: Modified kernel functions by geodesic distance. EURASIP J. Applied Signal Processing 16 (2004) 2515-2521

24. Gunn, S.: Support vector machines for classification and regression. Technical Report, School of Electronics and Computer Science, University of Southampton (1998)

25. Chen, Y., Wang, J.: Image categorization by learning and reasoning with regions. J. Machine Learning Research 5 (2004) 913-939

26. Deselaers, T., Keysers, D., Ney, H.: Features for image retrieval: A quantitative comparison. In: Proc. of 26th DAGM Symposium on Pattern Recognition. (2004)

27. Marée, R., Geurts, P., Piater, J., Wehenkel, L.: Random subwindows for robust image classification. In: Proc. of IEEE Computer Society Conf. Computer Vision and Pattern Recognition. (2005)

28. Liu, Q., Huang, R., Lu, H., Ma, S.: Face recognition using kernel based Fisher discriminant analysis. In: Proc. of Int. Conf. Automatic Face and Gestures Recognition. (2002) 